Article

\title{
Novel Video-Laryngoscope with Wireless Image Transmission via Wi-Fi towards a Smartphone
}

\author{
Mauricio Guerra-Hernández ${ }^{1, *}$, Gabriela Josefina Vidaña-Martínez ${ }^{2}$, José S. Camacho-Juárez ${ }^{3}$, \\ Hugo Barragán-Villegas ${ }^{2}$, José Enrique Calacuayo-Rojas ${ }^{3}$, Bersaín Alexander Reyes ${ }^{3}$ D, \\ Jorge Alberto Castañón-González ${ }^{4}$, Oscar Fernando Núñez-Olvera ${ }^{5}$ \\ and Ma. del Pilar Fonseca-Leal ${ }^{2}$ D \\ 1 Anestesiology Department, Hospital Central “Dr. Ignacio Morones Prieto", 78290 San Luis Potosi, Mexico \\ 2 Faculty of Medicine, Universidad Autonoma de San Luis Potosi, 78210 San Luis Potosi, Mexico; \\ gabyvida60@yahoo.com.mx (G.J.V.-M.); hbarraganixc@hotmail.com (H.B.-V.); \\ pilar.fonseca@uaslp.mx (M.d.P.F.-L.) \\ 3 Faculty of Science, Universidad Autonoma de San Luis Potosi, 78210 San Luis Potosi, Mexico; \\ sergio.camacho@uaslp.mx (J.S.C.-J.); enriquecalacuayo@gmail.com (J.E.C.-R.); \\ bersain.reyes@uaslp.mx (B.A.R.) \\ 4 Secretaría de Salud, Hospital Juarez de Mexico, Intensive Care and Critical Medicine Unit, \\ 07760 Mexico City, Mexico; jorge.castanong@gmail.com \\ 5 Instituto de Investigación en Comunicación Optica, Universidad Autonoma de San Luis Potosi, \\ 78210 San Luis Potosi, Mexico; oscar_n@cactus.iico.uaslp.mx \\ * Correspondence: mauricioguerrahdz@hotmail.com; Tel.: +52-444-8262300
}

Received: 2 September 2020; Accepted: 29 September 2020; Published: 3 October 2020

check for updates

\begin{abstract}
A low-cost video laryngoscope (VDL) called Hybrid 1.0 was developed using smart devices for visualization. To test its performance, we compared it with a high-end VDL device, using both in vitro and in vivo studies. During the in vitro study, medical students without experience in airway intubation were randomly asked to intubate a mannequin with different degrees of difficulty (Cormack-Lehane scales) by using either the Hybrid 1.0 VDL (GI) or a conventional laryngoscope (GII). During the in vivo study, $N=60$ endotracheal intubations were performed by resident and base physicians, divided into two groups; the first group intubated with the Hybrid 1.0 VDL (GI) while the second group used a VDL C-Mac shovel (GII). As performance indexes, both studies reported the number of successful intubations (correct capnography signal) and intubation time. For the in vitro testing, no statistically significant differences were found regarding the number of successful intubations, while statistically significant differences were found regarding the intubation times. During the in vivo tests, procedures were performed by residents and by base physicians, and no statistically significant differences were found. The provided results point out that the VDL proposed can be clinically useful and offers technical characteristics similar to other VDLs that currently exist on the market.
\end{abstract}

Keywords: video-laryngoscope; wireless; Wi-Fi; smartphone; endotracheal intubation

\section{Introduction}

Nowadays, the worldwide population is going through the coronavirus disease 2019 (COVID-19) pandemic generated by the severe acute respiratory syndrome coronavirus 2 (SARS-CoV-2). As a consequence, health professionals, companies and governments from all around the world have faced the fact that any medical equipment that is being used to treat this disease must not only be technically and clinically accepted but also affordable and accessible for any country [1]. In response to this emergency, many companies, universities and non-governmental organizations have developed 
mechanical ventilators to support respiratory disease patients [1-3]; however, even though medical staff expose their lives in such situations, only a few kinds of low-cost medical equipment have been presented to provide them with security during their clinical routine.

Clinically, the video laryngoscope (VDL) is a piece of medical equipment that supports intubating COVID-19 patients; its use is indicated in the guidelines for the treatment of such patients, as they provide a lower probability of contamination for medical staff who are in direct risk of contact with SARS-CoV-2 [4] It is well recognized that the use of VDLs has optimized the management of patients with difficult airways, and accordingly it has been included as part of the management of the airway guides of the American Society of Anesthesiologists (ASA) and the Difficult Airway Society (DAS) $[5,6]$. Furthermore, VDLs have had a great boom in developed countries, because they can help to better visualize the airways of patients who need to be intubated, since their use has shown great success in endotracheal intubation (EI) [7-10]. However, in developing countries, like ours, the approximate cost of VDL devices is between USD \$2,000 and USD \$22,000, while the cost of conventional laryngoscopes is within the range of USD $\$ 65$ to USD $\$ 110$. These limitations have contributed to the fact that it is still common to use conventional type laryngoscopes (CTLs) with Miller, Macintosh and McCoy type blades, where each one has its own advantage over the previous one: i.e., Miller helps to raise the tongue and assemble the epiglottis for better visualization; Macintosh is a leaf with a parabolic design capable of pulling the epiglottis from the vallecula and is more delicate with the tissues of the larynx; and the McCoy blade is able to bend over itself with an ingenious pulley mechanism that allows an angulation of the tip, improving the elevation of the epiglottis and visual field with a clearer appreciation of the glottis than in previous situations [11,12]. Although there are other blades, they are mainly derived from the first two.

Besides the benefits of CTLs with Miller and Macintosh blades, some difficulties arise. For example, with an intubation angle of 90 degrees, when intubating patients with morbid obesity and/or patients with too prominent a chest, usually the chest clogs the laryngoscope handle performing the EI [13]. Another problem arises with metallic shovels and the halogen light bulb, where heating of the shovel occurs after a few minutes of being on the luminous focal area, which could occur during difficult airway intubations and could lead to IE complications. Finally, it has been reported that a lack of visibility of the respiratory airways can happen while using CTLs [14].

In contrast to conventional laryngoscopes, VDLs usually do not present the abovementioned problems. However, they have higher costs than CTLs, mainly because of their more advanced instrumentation: e.g., they usually have special shovels and manufacturer-owned image display devices, with the associated costs of their consumables. In this paper, the development of a novel VDL based on a parabolic shovel is introduced. This geometry was proposed because it is natural to the shape of the respiratory airways. The proposed VDL was designed with a fixed angle of intubation of 110 degrees, LED lighting to visualize the airways and was built of medical grade resin. The novel VDL incorporates wireless communication that allows one to visualize the images in Wi-Fi (IEEE 802.11x protocol)-enabled devices such as smartphones, laptops or tablets. The design was made keeping in mind the need for an accessible cost in developing countries. Due to its ability to connect with commercially available smart devices, we named the proposed VDL system Hybrid 1.0. In addition to introducing the proposed system, a comparison of the probability of successfully intubating patients with a VDL Hybrid 1.0 was made with the one achieved while using a Macintosh CTL, particularly in cases where the physician has undergone an emergency EI and he/she has limited experience in performing such EI procedures.

\section{Materials and Methods}

\subsection{VDL System}

The developed Hybrid 1.0 VDL consists of three main components, as shown in Figure 1. The first component is a shovel racket with a parabolic geometry (Figure 1A) built from medical grade resin 
approved by the Food and Drugs Administration of the United States (177.2420-FDA). The handle has space to accommodate the video module (Figure 1, part A.1), and along the shovel there is space to house by pressure a video camera with autofocus and a resolution of $1270 \times 720$ pixels surrounded by light-emitting diodes (LEDs), as shown in Figure 1. The second component is the video module itself (Figure 1B), consisting of an Original Equipment Manufacturer (OEM) Wi-Fi video transmitter board with the commercial code YPC99 (Figure 1, part B.1). The last component is a mobile application (app) called Ycamera, which can be found in different digital application stores like the App Store and Google Play Store. This application is installed on a smartphone and allows the user to view the images transmitted via Wi-Fi (Figure 1C).
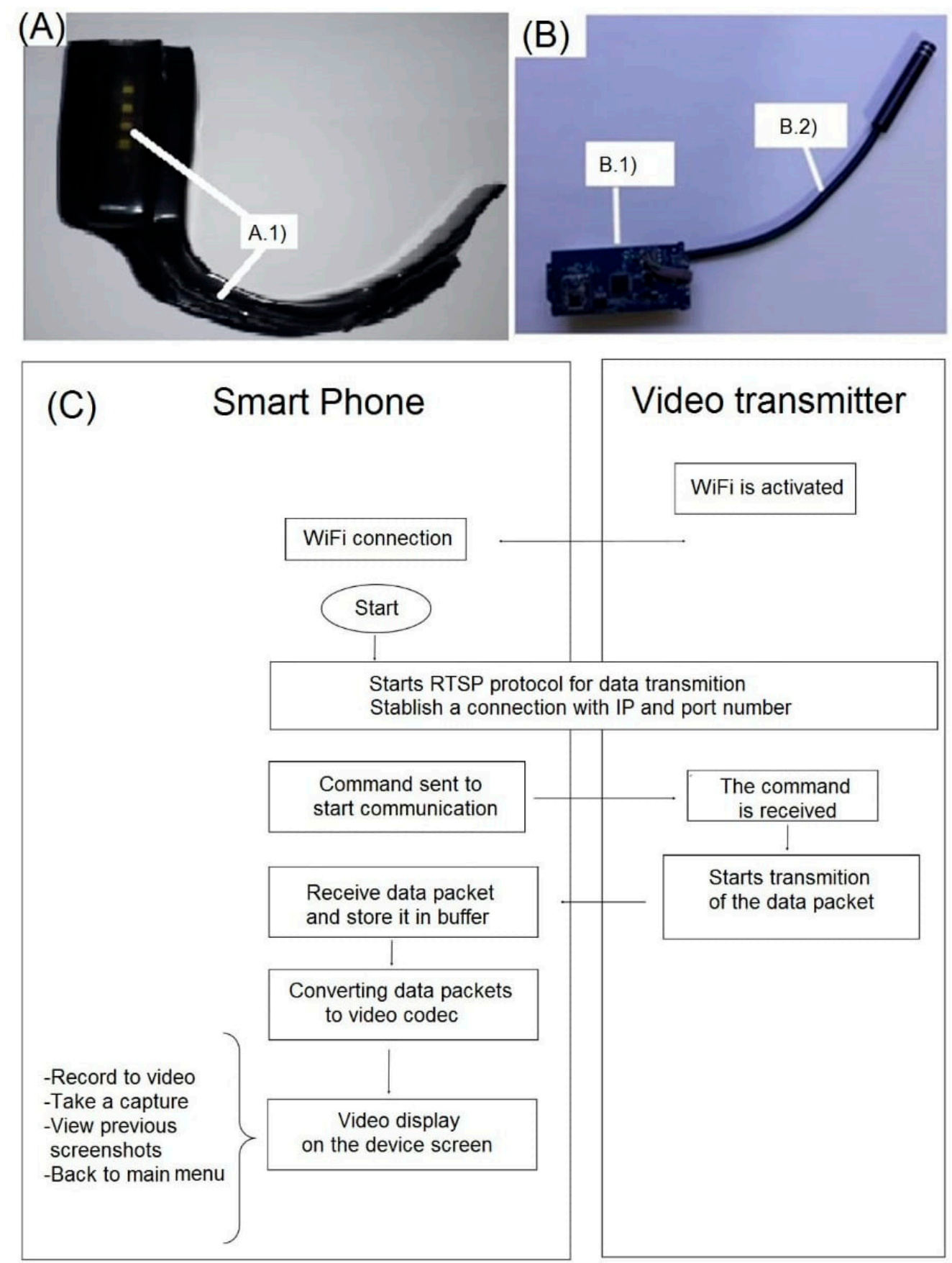

Figure 1. Main components of the developed Hybrid 1.0 video laryngoscope (VDL). (A) Shovel with parabolic geometry. (B) Wi-Fi video transmitter board. (C) Block diagram of the video transmission module and its wireless connection with a smartphone. 
The use of the proposed Hybrid 1.0 VDL is straightforward. First, once the application is installed on the smartphone, it should be verified that the video camera is well anchored to the VDL blade (Figure 1A). Then, the video module (Figure 1B) is turned on, followed by the turning on of the white LEDs. Finally, the video module will be connected to the smartphone app via the video transmitter (Figure 1C). Before inserting the VDL into the patient's mouth, the user must orient the camera towards a nearby object to verify that the images displayed on the app correspond to the focused object. Regarding the wireless video transmission, it works as shown in Figure 1C. First, once the video module is connected to the smartphone via Wi-Fi using a Realtek IEEE $802.11 \mathrm{~b} / \mathrm{g} / \mathrm{n} 2.4 \mathrm{GHz}$ wireless communication module, the real time streaming protocol (RTSP) is started. To this end, a client-server type communication is established between the smartphone and the video transmitter, by means of the Internet Protocol address and port number corresponding to the video transmitter. Then, a command is sent by the smartphone to initiate the transmission of data; the device receives the command and begins to send the video captured by the encoded camera in the form of data packets; these packets are received and stored in a buffer at a bit rate of $62.5 \mathrm{Mb} / \mathrm{s}$; then the data packets are decoded by the $\mathrm{H} 264$ video codec to be displayed on the smartphone screen. It is worth mentioning that the developed app incorporates functions to govern the video recording: i.e., to start/stop the recording, take a snapshot, visualize the previous snapshots and return to the main menu.

A block diagram of the main hardware components of the YPC99 Wi-Fi video transmitter board is shown in Figure 2. As can be seen, the VDL system is charged with a Fantassy brand (5V, 1A) wireless charger (Figure 2D) that sends an electromagnetic field to the wireless charging module embedded in the VDL (Figure 2E). The voltage that reaches the VDL is regulated by the wireless charger module and the VDL has a battery level indicator with part number IP- 5305 where four LEDs represents a $25 \%$ charge level with each LED when turned on. It takes about an hour to fully charge and about an hour to fully discharge if using it in a continuous fashion. Once the Hybrid 1.0 system is activated, the battery energizes the video camera and the Wi-Fi video transmitter board. The video camera can get and convert the images by means of a serial data transmission to the Wi-Fi video transmitter, which receives the data from the camera and manages the sending and receiving of data via the Wi-Fi transmitter towards the smartphone.

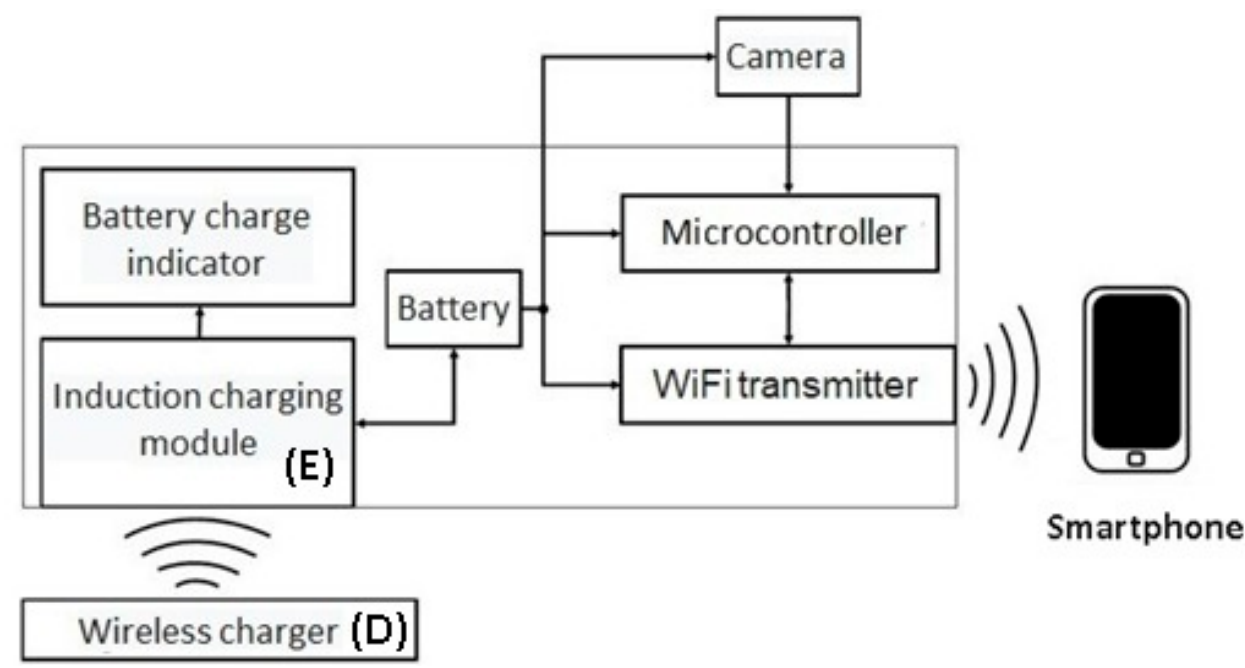

Figure 2. Block diagram of the charging and wireless communication with a smartphone.

\subsection{In Vitro Study with Training Mannequin to Simulate the Airway}

Before performing the in vitro and in vivo studies, force tests were performed on the Hybrid 1.0 VDL with a precision dynamometer of $20 \mathrm{~N}$ (3B Scientific, Hamburg, Germany) to measure the strength safely endured by the proposed VDL. To this end, the hook at the dynamometer's tip was anchored at the distal end of the VDL, then the dynamometer was stretched to the maximum length of the device. 
The in vitro study was performed as follows. First, $30 \mathrm{~s}$ year students from the Medical School of the Universidad Autonoma de San Luis Potosí were enrolled in this study. The enrolled students had no previous experience in airway intubation. All the students were made to intubate a mannequin Airway Management Trainer (Laerdal Brand, New York, NY, USA) with different Cormack scales. To this end, two groups of 15 students each were randomly created. The first group was required to intubate the mannequin with a VDL Hybrid 1.0 (GI) and the second group was required to intubate it with a conventional laryngoscope (Welch Allyn, Skaneateles Falls, NY, USA) with a Macintosh No. 3 shovel (GII). The mannequin was configured before performing the intubation and before a student was assigned to a given group. Finally, the number of successful intubations, given by a correct capnography signal, as well as the intubation time were registered.

\subsection{Clinical Tests in Patients}

\subsubsection{Subjects}

The study protocol was submitted for approval to the Committee of Ethics in Research and to the Research Committee of the Hospital Central Dr. Ignacio Morones Prieto (HCIMP) de San Luis Potosi, Mexico. The study was approved and assigned the registration number 20-19. The clinical procedure was cataloged with a risk greater than minimum. All the study procedures were made after signature of the informed consent letter by the patients. No images were recorded that could identify the patient and the registered data were kept anonymous.

The study was designed to account for sixty endotracheal intubations $(N=60)$ to be performed in the Anesthesiology Department of HCIMP by resident physicians of the second year $(\mathrm{R} 2=30)$, of the third year $(\mathrm{R} 3=23)$ and base physicians $(\mathrm{MB}=7)$, which were randomly divided into two groups as follows. The first group intubated patients with a VLG Hybrid 1.0 (GI), and the second group (GII) intubated patients with the VDL C-Mac shovel and D-Blade (Karl Storz, Tuttlingen, Germany), because the performed in vitro test showed that it could be dangerous to intubate with the LTC employed in such a test. The group of study volunteer patients were those requiring EI for surgery with general anesthesia. The inclusion criteria were patients scheduled for elective surgery who required general anesthesia or who underwent relative emergency procedures in which the management of the airway was the same as the one of a patient in elective surgery and who agreed to participate in the study by means of the informed consent. The demographic data of patients assigned to each group were as follow. GI age: $47 \pm 16$ years old (mean \pm standard deviation), 15 females and 15 males. GII age: $47 \pm 17.5$ years old, 19 female and 11 males. In both groups, patients were excluded in cases as follows: 1) during the preoperative assessment, the anesthesiologist determined that they were at risk of a difficult airway; 2) they were referred as having a difficult airway history in some previous surgery; or 3) during preoperative evaluation, the anesthesiologist determined that the patient required a rapid intubation sequence.

\subsubsection{Data Acquisition}

Measurements were performed after the pre-anesthetic and airway assessment, and only on those included patients who did not have more than three positive criteria for an airway difficulty assessment, as well as antecedents of airway difficulty. On the day of the procedure, the group (GI or GII) was randomly assigned to the physician performing the EI. During the procedure, the patient was placed in a decubitus supine posture and, after proper monitoring, the required anesthetic induction was performed. Then, the orotracheal intubation was performed using the conventional technique of intubation with a VDL. During the procedure, the visualized structures in the airway were recorded by means of the Cormack-Lehane scale. In addition, the ASA Classification, Mesh Scale and Bellhouse-Dore Classification were evaluated in each patient. 


\subsubsection{Statistical Analysis}

The statistical analysis was performed using the graphical user interface R Commander v.2.3 for the R software v.3.4.2 (the R Foundation), with a 95\% confidence level. The normality of the distribution of each group was assessed by means of the Shapiro-Wilk test. Continuous variables were reported as mean \pm S.D. or median (interquartile range) and (minimum-maximum), while discrete variables were reported as frequencies (\%). Finally, to evaluate the visualization of the larynx and glottis, in both groups, the mannequin configuration from the in vitro study and the patients from the clinical test who allowed a perfect visualization of such structures (Cormack 1 and 2) were grouped in GI, while those displaying problems (Cormack 3 and 4) were grouped in GII. Finally, depending on the distribution of the variables, the analysis was performed with a Student $t$ test or a Fisher test to check the primary objective; this one was that the intubation times in both the C-Mac and the VLG Hybrid 1.0 were similar.

\section{Results}

\subsection{In Vitro Study with Training Mannequin to Simulate the Airway}

Force tests of the VDL Hybrid 1.0, performed with a precision dynamometer, showed that the device resists more than $20 \mathrm{~N}$ without breaking.

Regarding the results of the in vitro test with the training mannequin, the Shapiro-Wilk test of intubation with a VDL Hybrid 1.0 (GI) and intubation with a Macintosh No. 3 shovel (GII) showed that each group had a distribution Shapiro-Wilk normality test, $\mathrm{W}=0.78043, \mathrm{p}=0.00002942$. The statistical results for the in vitro tests are shown in Table 1. The Fisher test results for GI were 13 successful intubations in 87 attempts and for GII were 9 successful intubations in 60 attempts. No statistically significant differences were found between GI and GII regarding successful intubations $(p=0.215)$. Regarding the intubation time, the medians were found to be equal to $\mathrm{Me}=27 \mathrm{~s}$ with an $\mathrm{IQR}=12.5 \mathrm{~s}$ for GI, and Me $=106 \mathrm{~s}$ with an IQR $=76 \mathrm{~s}$ for GII. In this case, statistically significant differences were found between the groups $(p=0.005)$.

Table 1. In vitro test results obtained during mannequin intubation for first group (GI) (intubation with 213 proposed video laryngoscope (VDL) Hybrid 1. 0) and second group (GII) (intubation with a Macintosh No. 3 shovel).

\begin{tabular}{|c|c|c|c|}
\hline Variables & $\begin{array}{c}\text { G I } \\
n=15(\%)\end{array}$ & $\begin{array}{c}\text { G II } \\
n=15(\%)\end{array}$ & $p$ Value \\
\hline Intubation time (s) & $\begin{array}{c}27 \\
{[21,33.5]} \\
(15-120)\end{array}$ & $\begin{array}{c}106 \\
{[44,120]} \\
(18-120)\end{array}$ & 0.005 \\
\hline Successful intubations & $13(87)$ & $9(60)$ & 0.215 \\
\hline Cormack scale & & & \multirow[b]{2}{*}{$<0.0001$} \\
\hline $\begin{array}{l}\text { One } \\
\text { Two } \\
\text { Three } \\
\text { Four }\end{array}$ & $\begin{array}{c}14(94) \\
1(6) \\
0 \\
0\end{array}$ & $\begin{array}{l}1(6) \\
8(54) \\
3(20) \\
3(20)\end{array}$ & \\
\hline Cormack groups & & & \multirow[b]{2}{*}{0.016} \\
\hline $\begin{array}{l}1=1 \text { y } 2 \\
2=3 \text { y } 4\end{array}$ & $\begin{array}{c}15(100) \\
0\end{array}$ & $\begin{array}{l}9(60) \\
6(40)\end{array}$ & \\
\hline
\end{tabular}

\subsection{Clinical Tests in Patients}

For the clinical tests in EI patients, it was found that the average age of the patients was 47 years old, 34 of the patents being women and 26 men. The ASA ratings were found to be ASA I (10\%), ASA II 
(55\%), ASA III (25\%) and ASA IV (10\%). The median intubation attempts were one, with a maximum of two attempts. It was also found that there were zero failed intubations, where $88 \%$ of the procedures were performed by residents and $22 \%$ by base physicians. The results of the clinical tests in EI patients are shown in Table 2.

Table 2. Clinical characteristics of endotracheal intubation patients and variables studied with 232 in each device group (GI: VLG Hybrid 1.0; GII: VDL C-Mac shovel and D-Blade).

\begin{tabular}{|c|c|c|c|c|}
\hline Variable & $N=60$ & $\begin{array}{c}\mathrm{GI} \\
n=30\end{array}$ & $\begin{array}{c}\text { GII } \\
n=30\end{array}$ & $\begin{array}{c}p \\
\text { Value }\end{array}$ \\
\hline Age (y.o.) & $\begin{array}{l}47 \pm 16 \\
(18-83)\end{array}$ & $\begin{array}{l}46 \pm 14 \\
(19-71)\end{array}$ & $\begin{array}{c}47 \pm 17.5 \\
(18-83)\end{array}$ & 0.78 \\
\hline Gender & & & & 0.434 \\
\hline Female & $34(57)$ & $15(50)$ & $19(63)$ & - \\
\hline Male & $26(43)$ & $15(50)$ & $11(37)$ & - \\
\hline ASA classification & & & & 0.77 \\
\hline ASA I & $6(10)$ & $3(10)$ & $3(10)$ & - \\
\hline ASA II & $33(55)$ & $17(57)$ & $16(53)$ & - \\
\hline ASA III & $15(25)$ & $6(20)$ & $9(30)$ & - \\
\hline ASA IV & $6(10)$ & $4(13)$ & $2(7)$ & - \\
\hline \multicolumn{5}{|l|}{ Cormack-Lehane scale } \\
\hline Grade 1 & $58(97)$ & - & - & - \\
\hline Grade 11 & $2(3)$ & - & - & - \\
\hline Grade 111 & 0 & - & - & - \\
\hline Grade 1V & 0 & - & - & - \\
\hline Mallampati scale & & & & 0.88 \\
\hline Class I & $19(32)$ & $10(33)$ & $9(30)$ & - \\
\hline Class II & $22(37)$ & $12(40)$ & $10(33)$ & - \\
\hline Class III & $15(25)$ & $6(20)$ & $9(30)$ & - \\
\hline Class IV & $4(7)$ & $2(7)$ & $2(7)$ & - \\
\hline \multicolumn{2}{|c|}{ Bellhouse-Dore classification } & & & 0.03 \\
\hline Grade 1 & $46(77)$ & $19(63)$ & $27(90)$ & - \\
\hline Grade 11 & $14(23)$ & $11(37)$ & $3(10)$ & - \\
\hline Grade 111 & 0 & 0 & 0 & - \\
\hline Intubation time (s) & $\begin{array}{l}24[11] \\
(16-79)\end{array}$ & - & - & - \\
\hline Number of attempts & $\begin{array}{c}1[0] \\
(1-2) \\
\end{array}$ & - & - & - \\
\hline Failed intubations & 0 & - & - & - \\
\hline Operator & - & - & - & - \\
\hline Resident 2 & $30(50)$ & - & - & - \\
\hline Resident 3 & $23(38)$ & - & - & - \\
\hline Base physician & $7(12)$ & - & - & - \\
\hline
\end{tabular}

To evaluate the performance of the devices (Table 3) the intubation time per group was measured, where the means were found to be $\mathrm{Me}=23.5 \mathrm{~s}$ and $\mathrm{Me}=26 \mathrm{~s}$ for GI and GII, respectively. No statistically significant differences were found regarding the intubation time between both groups $(p=0.136)$. The dispersion of the intubation times for both groups is presented in Figure 3 . In addition, no statistically significant differences were found regarding the number of attempts $(p=0.57)$, regarding the operator who performed the intubation $(p=0.08)$, as well as regarding the structure display according to the Cormack-Lehane scale $(p=0.49)$. Finally, examples of airway images obtained with the developed VDL Hybrid 1.0 and VDL C-Mac are shown in Figures 4 and 5, respectively. 
Table 3. Performance comparison of intubation with a VLG Hybrid 1.0 (GI) and with a VDL C-Mac (GII).

\begin{tabular}{lccc}
\hline \multicolumn{1}{c}{ Variable } & $\begin{array}{c}\text { GI } \\
n=30\end{array}$ & $\begin{array}{c}\text { GII } \\
n=30\end{array}$ & $\begin{array}{c}p \\
\text { Value }\end{array}$ \\
\hline Intubation time (s) & $23.5[8]$ & $26[14.5]$ & 0.136 \\
\hline Number of attempts & $(16-54)$ & $(17-79)$ & 0.57 \\
\hline Operator & $\begin{array}{c}1[0] \\
(1-2)\end{array}$ & $\begin{array}{c}1[0] \\
(1-2)\end{array}$ & 0.08 \\
\hline Resident 2 & & & - \\
Resident 3 & $12(40)$ & $18(60)$ & - \\
Base physician & $12(40)$ & $11(38)$ & 0.49 \\
\hline Cormack-Lehane scale & $6(20)$ & $1(3)$ & - \\
\hline Grade 1 & & & - \\
Grade 11 & $28(93)$ & $30(100)$ & - \\
Grade 1l1 & $2(7)$ & 0 & 0 \\
Grade 1V & 0 & 0 & - \\
\hline
\end{tabular}

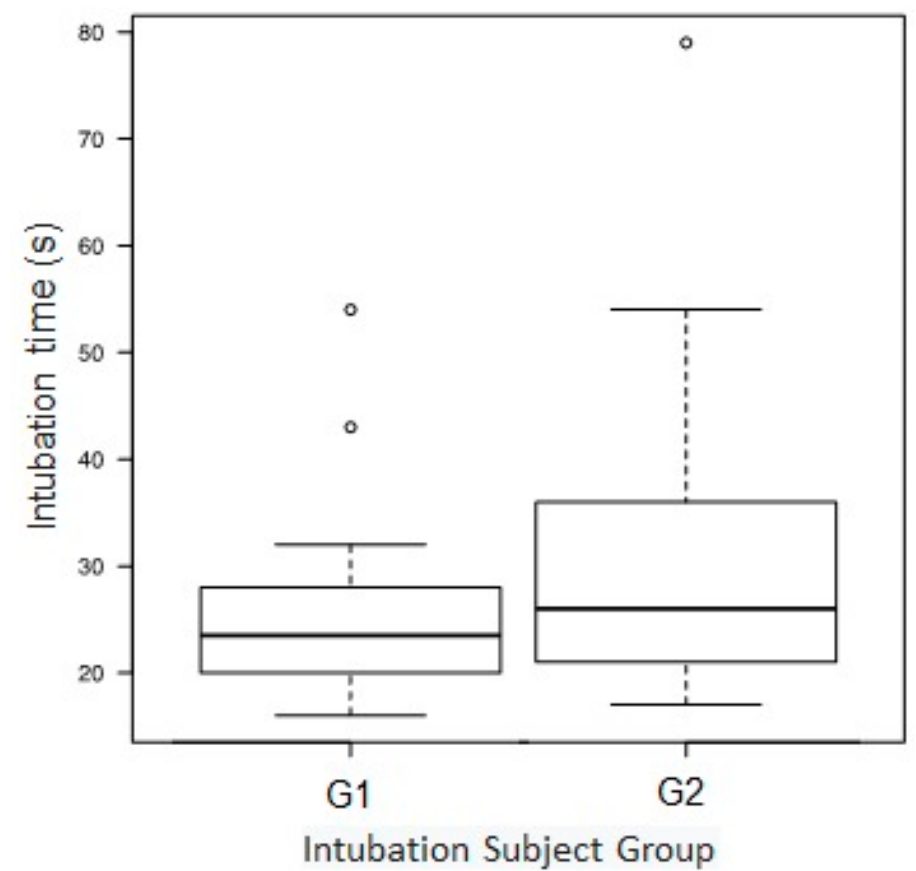

Figure 3. Time taken to perform a successful endotracheal intubation (EI) with VDL Hybrid 1.0 (first group (GI)) and with C-Mac (second group (GII)). 

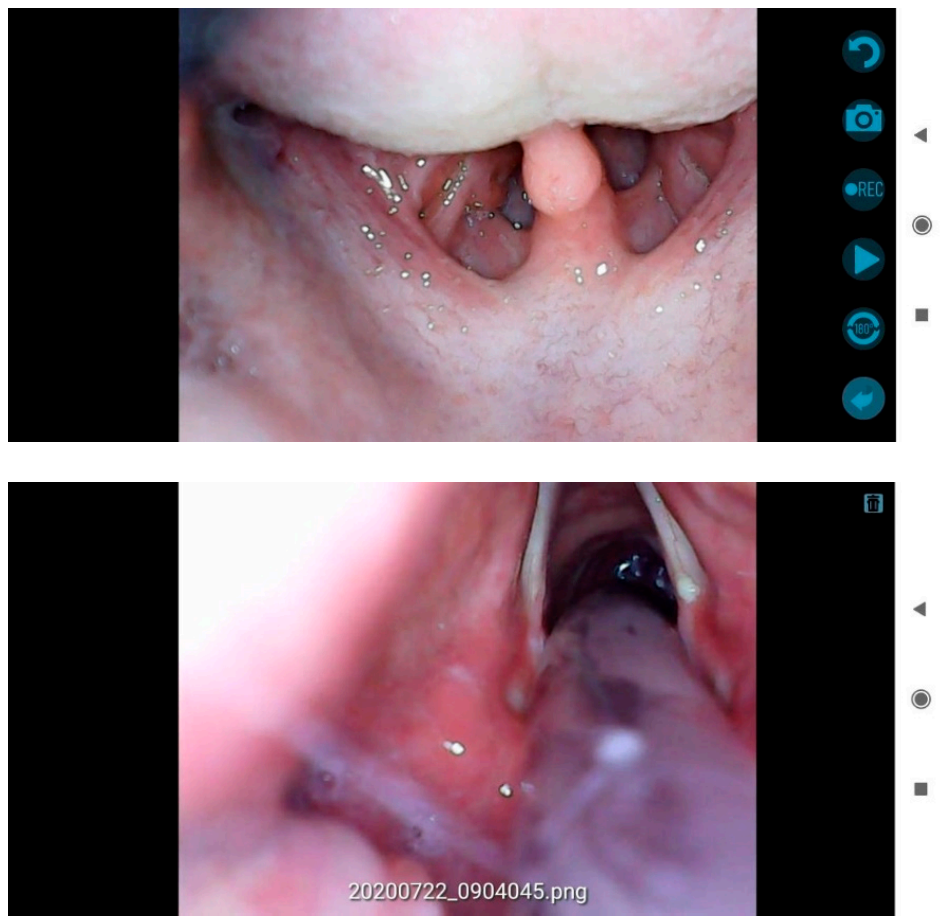

Figure 4. Example of app screenshots obtained with the proposed VDL Hybrid 1.0 during an endotracheal intubation in a real patient. Top: a screenshot while recording video. Bottom: a saved image using the app. The app activities are executed by selecting the blue buttons shown on the right edge of the screen.
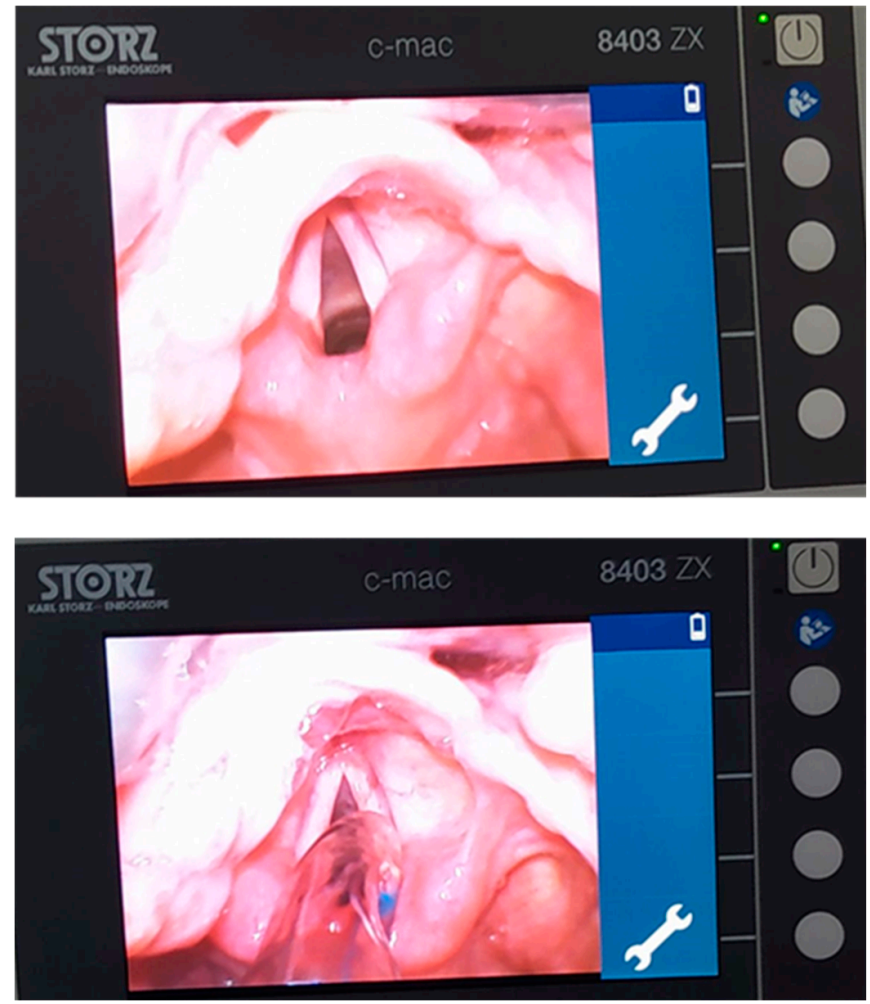

Figure 5. Similar example of VDL photographs obtained with C-Mac during an endotracheal intubation in a real patient. Top: photography during visualization of the buccal cords. Bottom: photograph of the introduction of the endotracheal cannula through the buccal cords. 


\section{Discussion}

Interestingly, in many privately owned companies that do not belong to the biomedical engineering field it is believed that the development of a medical device, e.g., a mechanical ventilator, is enough to market it [3]. Fortunately, according to the law, even to be able to test a medical device in patients it is necessary to meet certain requirements [15]. Therefore, in this study we addressed not only the development of a video laryngoscope, called Hybrid 1.0, but also its preliminary testing.

Although no pressure feedback is applied on the tongue due to the VDL Hybrid 1.0, a force test was performed to study if the proposed VDL supports enough force in case of accidents, assuring that no secondary damage is suffered by the patient. The results showed that the strength endured by the VDL Hybrid 1.0 was above $20 \mathrm{~N}$, which is higher than the one reported by Russell et al., who compared the force applied in performing direct laryngoscopy with that required for video laryngoscopy using the VDL GlideScope, and found that $9 \mathrm{~N}$ was the peak force required to do video laryngoscopy with the VDL Glidescope [16].

It is worth mentioning that at the beginning of the present work, the research group only aimed at developing a VDL that provides a better visualization of airway structures and thus allows EI to be performed faster and more successfully than with a conventional type laryngoscope. To assure a secure testing of the proposed VDL, an in vitro study was done by intubating a training mannequin with the proposed VDL Hybrid 1.0 and a CTL. The in vitro study results showed more successful intubations (13 successful intubations) were achieved while using the VDL Hybrid 1.0 than while using the CTL (9 successful intubations). In addition, the median intubation time while using the VDL Hybrid 1.0 $(\mathrm{Me}=27 \mathrm{~s})$ was found to be approximately four times lower $(27 \mathrm{~s} / 106 \mathrm{~s}=0.255)$ than the corresponding one while employing a CTL. Even though the number of EI attempts was higher while using the VDL Hybrid 1.0 (87) than while using the CTL (60), the results appear to indicate that it was due to the number of times that a EI was performed to the mannequin with both devices, because on average the number of EIs achieved with the VDL Hybrid 1.0 (87 successful intubations/13 intubated patients $=6.69$ successful intubations/intubated patients) is approximately the same as the average of EIs achieved with the CTL ( 60 successful intubations/9 intubated patients $=6.66$ successful intubations/intubated patients). We considered that a drawback could arise if one considers the median intubation time as the representative index for all physicians that were able to intubate the mannequin and divided this amount by the average successful intubations/intubated patients. In the case of the VDL Hybrid 1.0, the time per intubation would be $4.03 \mathrm{~s} /$ intubation $(27 \mathrm{~s} / 6.69$ intubations), which is four times shorter than while intubating with the CTL (4.03 s/intubation), which shows that it is four times shorter than IE by means of the LTC that would be equal to $15.9 \mathrm{~s} /$ intubation (106 s/6.66 intubations). Therefore, in this study we considered it dangerous to perform the clinical tests with a CTL; then, the performance of proposed VDL for EI in real patients was compared against the VDL Karl Storz model C-Mac.

Regarding the clinical study, we found that the age of both groups was very similar (GI: $46 \pm 4$ years old, and GII: $47 \pm 17.5$ years old). In addition, the ASA classification in both groups was the same (100 attempts/group), and the highest class of EI attempts occurred when the patients had an ASA II surgical risk classification. Another scale used to intubate is the Mallampati scale; the results showed that Class I was very similar in both groups (GI: 33, GII: 30). The Bellhose-Dore scale, which allows physicians to know about cervical mobility in order to move the head to certain angles, showed that the percentage of patients who could place the head at an angle greater than 35 degrees was higher for GII (GI: $63 \%$, GII: $90 \%$ ), and the percentage of patients who were able to move the head to $1 / 3$ or between $12^{\circ}$ and $35^{\circ}$ degrees was higher for GI (GI: $37 \%$, GII: $10 \%$ ).

Regarding the performance evaluation of VDLs for the intubation time $(p=0.136)$, for the number of attempts $(p=0.57)$, for the operator performing the EI $(p=0.08)$ and for the adequate visualization according to the Cormack-Lehane scale $(p=0.49)$, no statistically significant differences were found in any of those parameters. We consider that these results are due to the physical characteristics exhibited by both devices and which are discussed below. 
Although the median of endotracheal intubation time was found to be approximately equal in both groups, GI: 23.5 s and GII: 26 s., we found a lower dispersion in GI, as shown in Figure 3. Even though the VDL C-Mac has good video quality compared to the VDL Hybrid 1.0, we consider that this behavior in the EI time could be attributed to the wire that connects the former to its central processing unit: i.e., the VDL C-Mac is heavier (C-Mac blade weight $=320 \mathrm{~g})$. In contrast, the proposed VDL is wireless and lighter (VDL Hibryd 1.0 weight $=110 \mathrm{~g}$ ), resulting in its being easier to operate. Therefore, we consider that the disadvantage of the device in video quality is compensated by the disadvantage of the other VDL in terms of usability. The results for the degree of visualization of laryngeal structures, according to the Cormack-Lehane scale, showed that the VDL C-Mac exhibited a superior performance than that of the proposed VDL Hybrid 1.0 (GI: Cormack grade I = 93, GII: Cormack grade I: 100). However, that advantage was found to be not statistically significant.

It is worth mentioning that during the clinical procedures, on five occasions (three with the Hybrid 1.0 and two with the C-Mac laryngoscope video) it was difficult to introduce the endotracheal tube posterior to the vocal cords passage, despite the clear visualization of the laryngeal structures in the VDLs. This was probably because of an increased leaf angulation that resulted in a poor rectification between thyroid cartilage and cricoid, generating a sharp step where the endotracheal tube stops. This particularity was successfully corrected by slightly releasing the traction on the handle to recover the patient's own anatomical angulation.

Finally, this paper presented a novel VDL, the so-called Hybrid 1.0, which is light, reusable and wireless, and in turn frees the VDL from relying on a specific external monitor, making it cheap compared to the commercially available ones in our country, with a projected price of approximately USD \$1000 to USD \$1500. We consider that such technical and economical features must be satisfied by medical devices nowadays to support nations in the event of contingencies such as the pandemic COVID-19 currently affecting our planet.

\section{Conclusions}

The proposed VDL Hybrid 1.0 is a device that allows adequate performance of endotracheal intubations, by offering an intubation time similar to the VDL C-Mac, as well as with an adequate visualization in terms of the quality and quantity of the anatomical structures involved in the EI procedure.

The results provided in this study appear to indicate that the developed VDL Hybrid 1.0 is a clinically useful, portable, wireless device. Besides its significantly lower cost, it offers technical characteristics very similar to other VDLs currently available on the market. Finally, we consider that the Hybrid 1.0 can be considered as a support device not only in the operating room, but in extra-hospital environments, emergency rooms and other units where advanced airway management is required.

Author Contributions: The individual contributions of each of the authors to this research article were: Conceptualization, M.G.-H.; methodology G.J.V.-M., H.B.-V. and J.A.C.-G.; software, J.E.C.-R.; validation, M.G.-H., G.J.V.-M., H.B.V. and M.d.P.F.-L.; formal analysis, M.d.P.F.-L.; investigation, M.G.H.; resources, J.E.C.-R.; data curation, M.D.P.F.-L.; writing - original draft preparation, J.S.C.-J., B.A.R., O.F.N.-O. and J.A.C.-G.; writing—review and editing, J.S.C.-J., B.A.R. and O.F.N.-O.; visualization, B.A.R. All authors have read and agreed to the published version of the manuscript.

Funding: This research received no external funding.

Conflicts of Interest: Authors J. S. C. J., J. E. C. R. and M. G. H. are pursuing an international patent for the video laryngoscope Hybrid 1.0. 


\section{References}

1. MIT-Based Team Works on Rapid Deployment of Open-Source, Low-Cost Ventilator, MIT News. Available online: http://news.mit.edu/2020/ventilator-covid-deployment-open-source-low-cost-0326 (accessed on 27 July 2020).

2. Istituto Italiano di Tecnologia. FI5 Pulmonary Ventilator. Available online: https://www.iit.it/iit-vs-covid-19/ fi5-ventilator (accessed on 27 July 2020).

3. From Making Cars to Ventilators. Available online: https://www.volkswagenag.com/en/news/stories/2020/ 04/from-making-cars-to-ventilators.html (accessed on 27 July 2020).

4. Colegio Mexicano de Medicina Crítica. Guía COVID-19 para la atención del paciente crítico con infección por SARS-coV-2 (segunda parte). Med. Crítica 2020, 34, 99-124. [CrossRef]

5. Apfelbaum, J.L.; Hagberg, C.A.; Caplan, R.A.; Blitt, C.D.; Connis, R.T.; Nickinovich, D.G. Practice guidelines for management of the difficult airway: An updated report by the American Society of Anesthesiologists Task Force on Management of the Difficult Airway. Anesthesiology 2013, 118, 251-270. [CrossRef] [PubMed]

6. Frerk, C.; Mitchell, V.S.; McNarry, A.F.; Mendonca, C.; Bhagrath, R.; Patel, A.A.; O'Sullivan, E.P.; Woodall, N.M.; Ahmad, I. Difficult Airway Society 2015 guidelines for management of unanticipated difficult intubation in adults. BJA Br. J. Anaesth. 2015, 115, 827-848. [CrossRef] [PubMed]

7. Hoshijima, H.; Kuratani, N.; Hirabayashi, Y.; Takeuchi, R.; Shiga, T.; Masaki, E. Pentax Airway Scope ${ }^{\circledR}$ vs Macintosh laryngoscope for tracheal intubation in adult patients: A systematic review and meta-analysis. Anaesthesia 2014, 69, 911-918. [CrossRef] [PubMed]

8. Smereka, J.; Ladny, J.R.; Naylor, A.; Ruetzler, K.; Szarpak, L. C-MAC compared with direct laryngoscopy for intubation in patients with cervical spine immobilization: A manikin trial. Am. J. Emerg. Med. 2017, 35, 1142-1146. [CrossRef] [PubMed]

9. Saricicek, V.; Mizrak, A.; Gul, R.; Goksu, S.; Cesur, M. GlideScope video laryngoscopy use tracheal intubation in patients with ankylosing spondylitis: A series of four cases and literature review. J. Clin. Monit. Comput. 2014, 28, 169-172. [CrossRef] [PubMed]

10. Hazarika, H.; Saxena, A.; Meshram, P.; Bhargava, A.K. A randomized controlled trial comparing C Mac D Blade and Macintosh laryngoscope for nasotracheal intubation in patients undergoing surgeries for head and neck cancer. Saudi J. Anaesth. 2018, 12, 35-41. [CrossRef] [PubMed]

11. Bailey, B. Laryngoscopy and laryngoscopes-Who's first?: The forefathers/four fathers of laryngology. Laryngoscope 1996, 106, 939-943. [CrossRef] [PubMed]

12. McCoy, E.P.; Mirakhur, R.K. The levering laryngoscope. Anaesthesia 1993, 48, 516-519. [CrossRef] [PubMed]

13. Lewis, S.R.; Butler, A.R.; Parker, J.; Cook, T.M.; Smith, A.F. Videolaryngoscopy versus direct laryngoscopy for adult patients requiring tracheal intubation. Cochrane Database Syst. Rev. 2016, 11, 15. [CrossRef] [PubMed]

14. Bustillos-Gaytán, M.L.; Palacios-Ríos, D.; López-Cabrera, N.G.; Rodríguez-Flores, A.M.; García-Torres, R.M.; Mendoza-Rosales, C.F. Effectiveness of a laryngoscope blade with video adaptation. Anest. México 2018, 30, 28-35.

15. Reglamento de la Ley General de Salud en Materia de Investigación para la Salud. Available online: http://www.salud.gob.mx/unidades/cdi/nom/compi/rlgsmis.html (accessed on 13 August 2020).

16. Russell, T.; Khan, S.; Elman, J.; Katznelson, R.; Cooper, R.M. Measurement of forces applied during Macintosh direct laryngoscopy compared with GlideScope ${ }^{\circledR}$ videolaryngoscopy. Anaesthesia 2012, 67, 626-631. [CrossRef] [PubMed]

(C) 2020 by the authors. Licensee MDPI, Basel, Switzerland. This article is an open access article distributed under the terms and conditions of the Creative Commons Attribution (CC BY) license (http://creativecommons.org/licenses/by/4.0/). 